\title{
Using Connected Vehicle Data to Reassess Dilemma Zone Performance of Heavy Vehicles
}

Howell Li, Corresponding Author

Purdue University

207 S Martin Jischke Dr., West Lafayette, IN 47907

Phone: 765-494-9601

Email: howell-li@purdue.edu

\section{Tom Platte}

Indiana Department of Transportation

$8620 \mathrm{E} 21^{\text {st }}$ St, Indianapolis, IN 46350

Phone: 317-402-4459

Email: toplatte@indot.in.gov

\section{Jijo Mathew}

Purdue University

207 S Martin Jischke Dr., West Lafayette, IN 47907

Phone: 765-494-4521

Email: kjijo@purdue.edu

\section{W. Benjamin Smith}

Indiana Department of Transportation

279 W 300 N, Crawfordsville, IN 47933

Phone: 765-361-5668

Email: wsmith1@indot.in.gov

\section{Enrique Saldivar-Carranza}

Purdue University

207 S Martin Jischke Dr., West Lafayette, IN 47907

Phone: +52 1 333-454-7268

Email: esaldiva@purdue.edu

\section{Darcy M. Bullock}

Purdue University

207 S Martin Jischke Dr, West Lafayette, IN 47907

Phone: 765-496-2226

Email: darcy@purdue.edu

July 30, 2019 


\begin{abstract}
The rate of fatalities at signalized intersections involving heavy vehicles is nearly five times higher than for passenger vehicles. Previous studies have found that heavy vehicles are twice as likely to violate a red light compared to passenger vehicles. Current technologies leverage setback detection to extend green time for a particular phase and are based upon typical deceleration rates for passenger cars. Furthermore, dilemma zone detectors are not effective when the max out time expires and forces the onset of yellow. This study proposes the use of Connected Vehicle (CV) technology to trigger Force Gap Out (FGO) before a vehicle is expected to arrive within the dilemma zone limit at max out time. The method leverages position data from Basic Safety Messages (BSMs) to map-match virtual waypoints located up to 1,050ft in advance of the stop bar. For a 55 miles per hour ( $\mathrm{mph}$ ) approach, field tests determined that using a $6 \mathrm{ft}$ waypoint radius at 50ft spacings would be sufficient to match $95 \%$ of BSM data within a 5\% lag threshold of 0.59s. The study estimates that FGOs reduce dilemma zone incursions by $34 \%$ for one approach and had no impact for the other. For both approaches, the total dilemma zone incursions decreased from 310 to 225. Although virtual waypoints were used for evaluating FGO, the study concludes by recommending that trajectory-based processing logic be incorporated into controllers for more robust support of dilemma zone and other emerging CV applications.
\end{abstract}

Keywords: connected vehicles (CV), dedicated short range communication (DSRC), dilemma zone, automated traffic signal performance measures, high-resolution controller event data, green extension, heavy trucks, probability of stopping 


\section{INTRODUCTION}

According to FHWA, signalized intersection fatalities account for approximately $27 \%$ of all total traffic fatalities (1) and of those, about 31\% involve heavy vehicles (2). In 2018 in Indiana, the rate of fatal crashes involving heavy vehicles at signalized intersections is nearly five times higher than crashes that do not involve a heavy vehicle (3). Previous studies have found that for red light violations, heavy vehicles entered the intersection later than passenger vehicles after the end of the yellow $(4,5)$, and were twice as likely to violate the red light than passenger vehicles $(5-7)$. They also require substantially longer stopping distance compared to passenger vehicles due to air brake lag and braking performance differences (7).

The stop-or-go decision is made at the onset of yellow, but at the dilemma zone a vehicle can neither comfortably stop nor clear the intersection $(6,8-11)$. Past studies have found increasing the yellow time to be effective for mitigating the number of red-light violations $(12,13)$. However, drivers tend to adapt to increased yellow times resulting in lower probabilities of stopping (12). Different dilemma zone boundaries for heavy vehicles and passenger vehicles would also require reconciling yellow timing objectives for balancing efficiency and safety (13).

In isolated, fully-actuated high-speed rural intersections, another solution is to use green extension. Studies have found that approaches with green extension systems reduced the number of red-light violations, hard braking or other evasive actions $(6,12,13)$. While green extensions are not visually detectable by the driver, one study found that drivers were less likely to stop due to adapted expectation compared to fixed-time systems (14). Since the benefit of green extension comes mainly from reducing the exposure of vehicles to the onset of yellow (14), when there is conflicting demand and the maximum green time has been reached (max out), the phase must inevitably terminate and any safety benefits are negated $(15,16)$. As the time to max out approaches, the dilemma zone protection boundary decreases (17). Sharma et al. implemented an approach to reduce max outs by selectively turning off detection in anticipation of future demand to reduce the number of green extensions. The study had found modified simultaneous gap out logic to reduce dilemma zone incursions by a quarter (16).

\section{MOTIVATION}

Studies have demonstrated that extending detection further upstream of an intersection can result in reductions in dilemma zone incursions $(4,7,18)$. Using camera or radar-based wide-area detectors can also mitigate dilemma zones and improve efficiency and safety at deployed intersections $(19,20)$, but these require sensor calibration.

\section{OPPORTUNITIES FOR IMPROVED DILEMMA ZONE DETECTION}

Connected vehicle (CV) technology using Dedicated Short Range Communications (DSRC) protocols can now detect equipped vehicles from about $1000 \mathrm{ft}$ to $1.2 \mathrm{miles}$ by a road-side unit (RSU) $(21,22)$ and shows good potential to prevent dilemma zone incursions. The Basic Safety Messages (BSMs) transmitted by the vehicles to the intersection contain latitude and longitude, speed, elevation, heading, braking information, and timestamp at $0.1 \mathrm{~s}$ interval (23). Recent research has explored using CV to mitigate dilemma zone incursions in simulation (24-28), but there have been limited field studies done on the topic thus far. This study investigates the use of map-matching of BSMs to virtual waypoints in lanes upstream of a signalized intersection to 
determine vehicle position relative to the stop bar. If dilemma zone mitigation is necessary, phase control action using NTCIP 1202 objects is initiated (29).

\section{OBJECTIVES}

The objective of this study is 1) to assess whether matching BSMs to virtual waypoints provides sufficient performance for dilemma zone mitigating tactics, 2) develop a dilemma zone mitigating tactic for CV, and 3) evaluate the performance of the tactic using Automated Traffic Signal Performance Measures (ATSPM) data.

\section{STUDY LOCATION AND INSTRUMENTATION}

A rural high-speed signalized intersection in Indiana is selected for the field study, shown in Figure 1a. The mainline arterial US-231 has $12 \mathrm{ft}$ lanes and runs north-south and with speed limit of $55 \mathrm{mph}$. The mode distribution is $26 \%$ heavy vehicles. Loop detectors are located $405 \mathrm{ft}$ upstream of the stop bar in both of the mainline approaches (callout $\mathrm{i}$, callout ii) with a $5 \mathrm{~s}$ vehicle extension time. The intersection runs fully actuated with a 60 s max time on the mainline phases. Tire marks indicate this location may have been subjected to possible dilemma zone incursions (Figure 1b).

An RSU is instrumented atop the northeast pole facing southwest (Figure 1c, callout iii) and is connected to the same subnet as the traffic signal controller. Any BSM received is immediately forwarded to an embedded co-processing unit within the controller where the map-matching is performed. A series of 22 virtual waypoints with $50 \mathrm{ft}$ spacing in the main through lanes are defined starting from the stop bar up to 1,050 feet (Figure 1a, callout iv).

\section{DATA USED FOR ANALYZING DILEMMA ZONE PERFORMANCE}

The development of ATSPMs in the late 2000s have enabled agencies to modernize the way traffic signal management is performed (30-32). Agencies have been able to make performance-based, data-driven decisions to promote safety, efficiency, and equity when managing and operating their traffic signals (33). In particular to dilemma zones, ATSPM data records the onset of yellow time and whether a phase termination is due to a gap out, max out, or force off at $0.1 \mathrm{~s}$ interval (32). Additionally, vehicle detection data is recorded by channel and can be compared to the phase termination time to determine whether a vehicle was in the dilemma zone at the onset of yellow. All other phase transitions triggered by the controller are also recorded. 


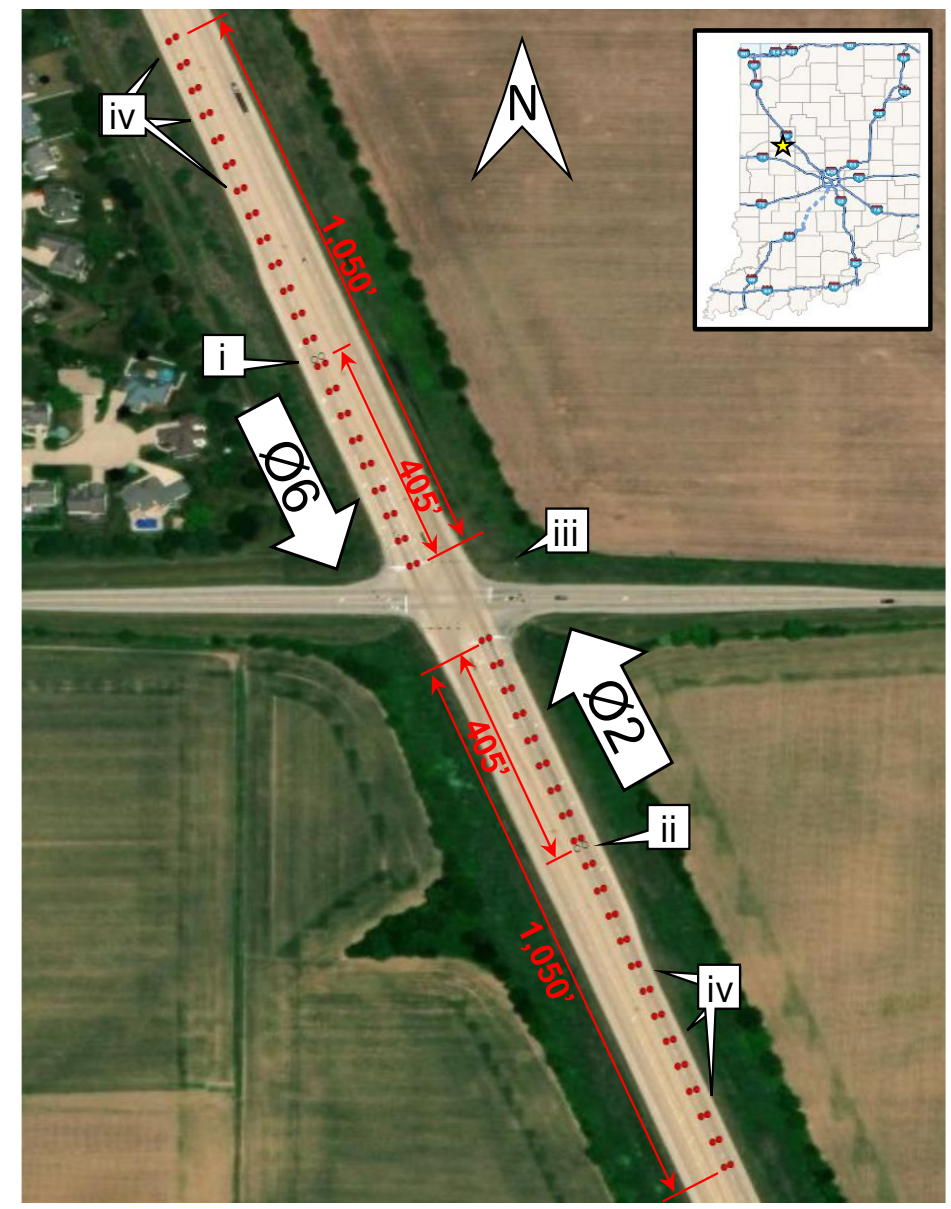

a. Intersection overview.

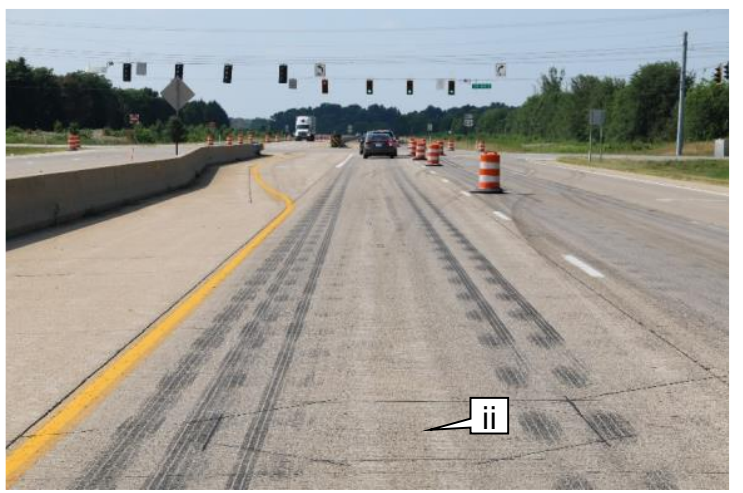

b. Northbound approach.

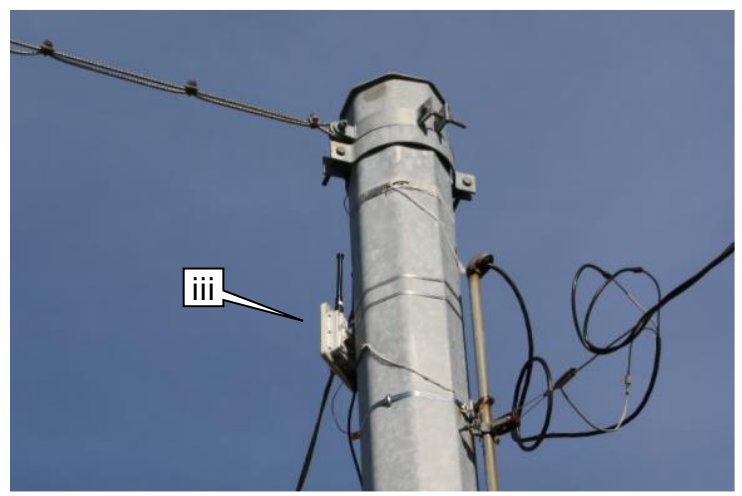

c. Radio mounting scheme.

Figure 1. Study location. 


\section{METHODOLOGY}

\section{Architecture}

A vehicle instrumented with a CV onboard unit (OBU) is used for sending BSMs to the RSU. The RSU forwards received packets above a threshold of $-82 \mathrm{dbm}$ to the embedded co-processor unit installed within the traffic signal controller. A set of virtual waypoints containing latitude, longitude, a range of acceptable heading, and associated lane and phase information is preloaded on the co-processor, where an application persistently listens for new BSMs. Each received packet within the acceptable strength threshold is decoded and matched to the set of virtual waypoints. If the vehicle sending the BSM is in proximity of a waypoint within the range of acceptable heading, a call is placed via NTCIP for the associated phase. All BSMs, successful waypoint matches, and phase calls are logged locally on the co-processor. In addition, the BSMs are uploaded to the backoffice via virtual private network (VPN) where it is stored using Apache Kafka, a fast stream processing platform capable of handling large volumes of data for future scalability (34). A web application then retrieves data from the platform where vehicle position and properties are displayed on a user interface during testing.

According to the specification for MAP messages in the SAE J2735 message definition, lane node information is defined as a set of latitude and longitude points (23). The methodology for vehicleto-lane position matching in this study is therefore designed to use point-to-point distance computation metric (35).

\section{Evaluating and selecting waypoint radius}

The radius is the maximum distance from each waypoint that a vehicle can be matched. Figure $2 \mathrm{a}$ illustrates a series of radii and hypothetical number of matches depending on a vehicle's speed. With a transmit interval of $0.1 \mathrm{~s}$, a vehicle travelling at $55 \mathrm{mph}(80.7 \mathrm{fps})$ is not guaranteed to match a waypoint with a $3 \mathrm{ft}$ radius threshold (callout i), assuming the vehicle's trajectory, OBU antenna, and waypoint are all reasonably centered in the lane.

Figure $2 \mathrm{~b}$ through e show trial runs at about $45 \mathrm{mph}$ (excluding stopping for red) for each of the radii listed in Figure 2a for 50ft spacings. Out of the 22 waypoints, a $3 \mathrm{ft}$ radius threshold had 16 out of the 22 waypoints missed (Figure $2 \mathrm{~b}$, red dotted lines), while radii $6 \mathrm{ft}$ and greater had no missed waypoints (dark crosses). The larger radii of $9 \mathrm{ft}$ and $12 \mathrm{ft}$ yielded more matches per waypoint, but risk encroachment into adjacent lanes. If more than one waypoint was matched, the waypoint closest to the vehicle was selected, which performed well to exclude adjacent lanes. For this study, a $6 \mathrm{ft}$ radius threshold is used which covers one lane width at the study location.

\section{Evaluating and selecting waypoint spacing}

The spacing is the distance between the centers of two consecutive waypoints. As the spacing increases, the lag between matches also increases because the vehicle needs to "traverse the gap." Figure 3a illustrates the time lag for a pair of waypoints with a $6 \mathrm{ft}$ radius for a series of spacings. For a $12 \mathrm{ft}$ spacing, every BSM would be matched assuming vehicle and waypoints are both centered in the lane, thus the lag is constantly $0.1 \mathrm{~s}$. The tradeoff for densely populating waypoints is that the size of the MAP message will substantially increase and may cause capacity challenges under load. The lag stays below $1 \mathrm{~s}$ for spacings of $75 \mathrm{ft}, 50 \mathrm{ft}$, and $25 \mathrm{ft}$. 


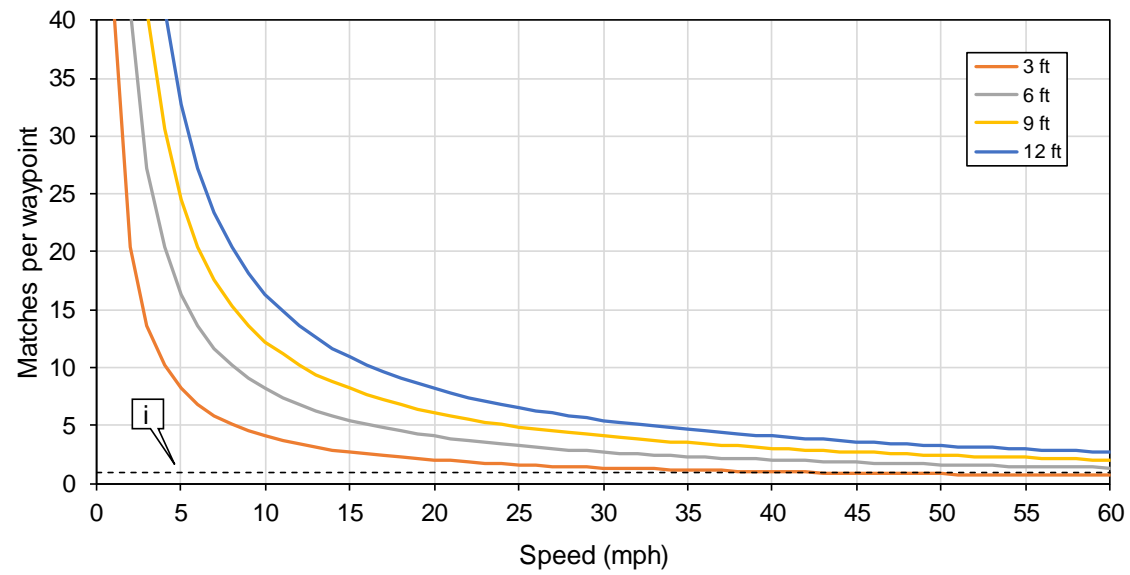

a. Hypothetical.
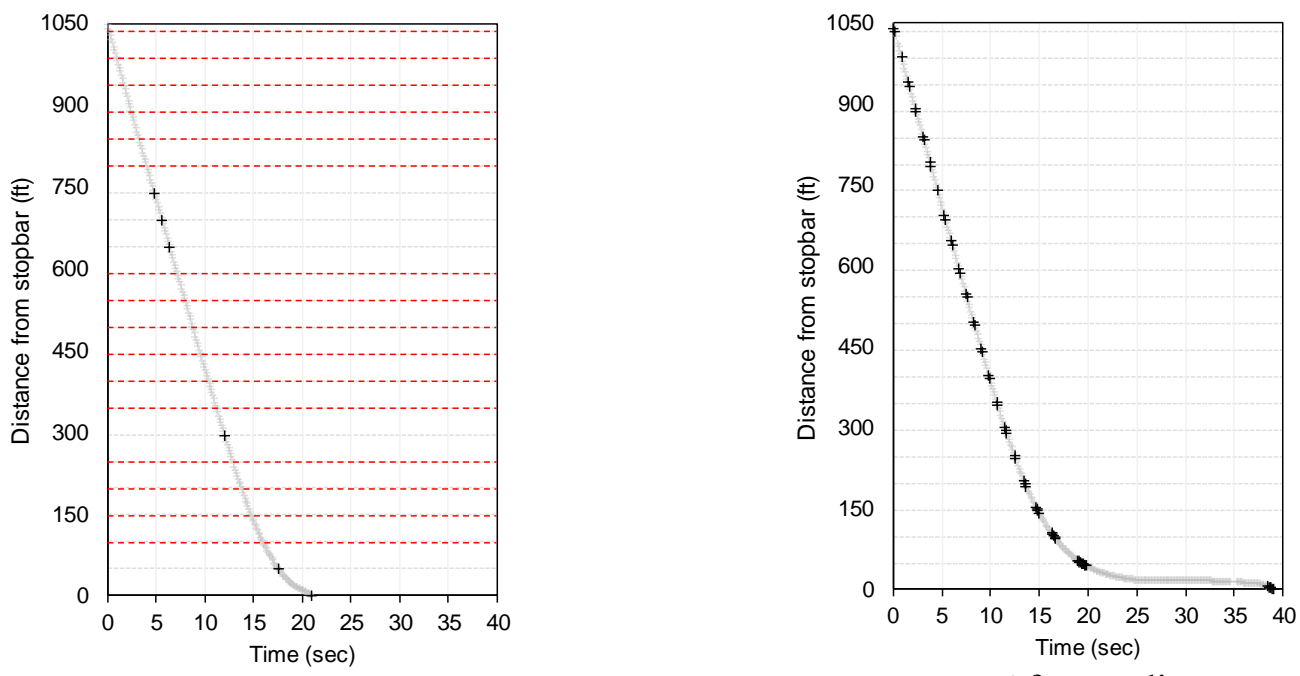

b. 3 foot radius.
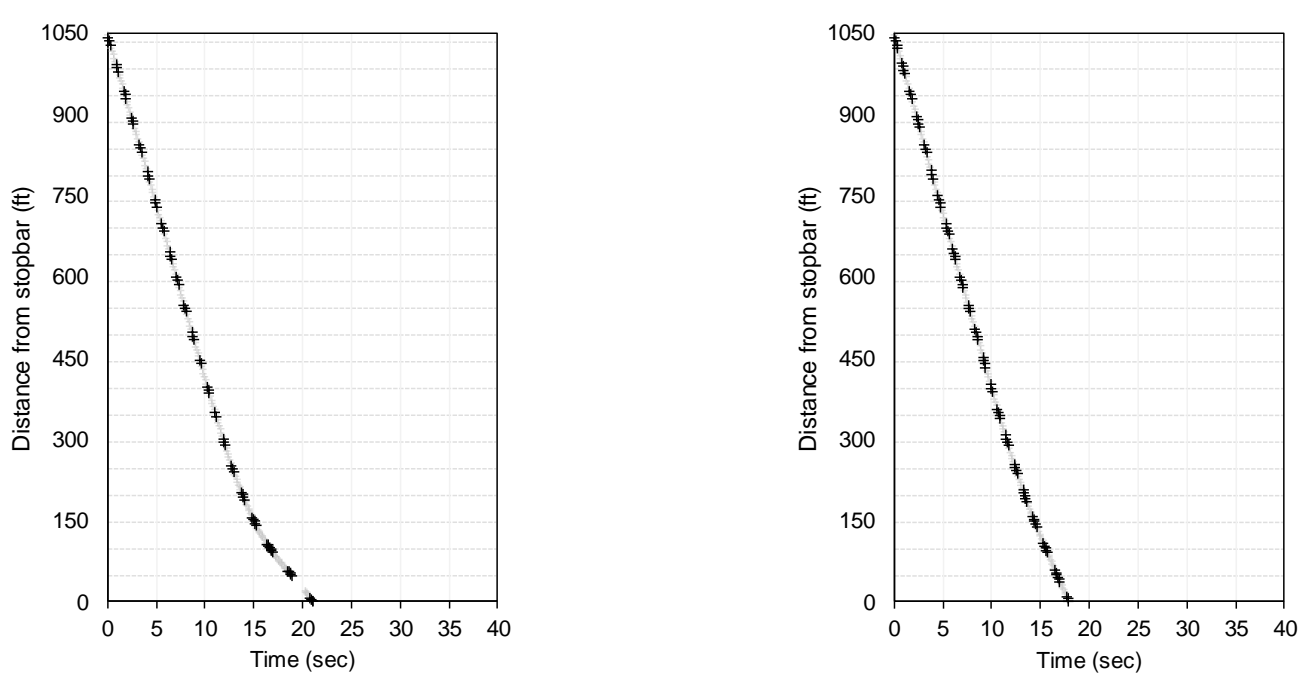

d. 9 foot radius.

e. 12 foot radius.

Figure 2. Performance of different waypoint radii. 


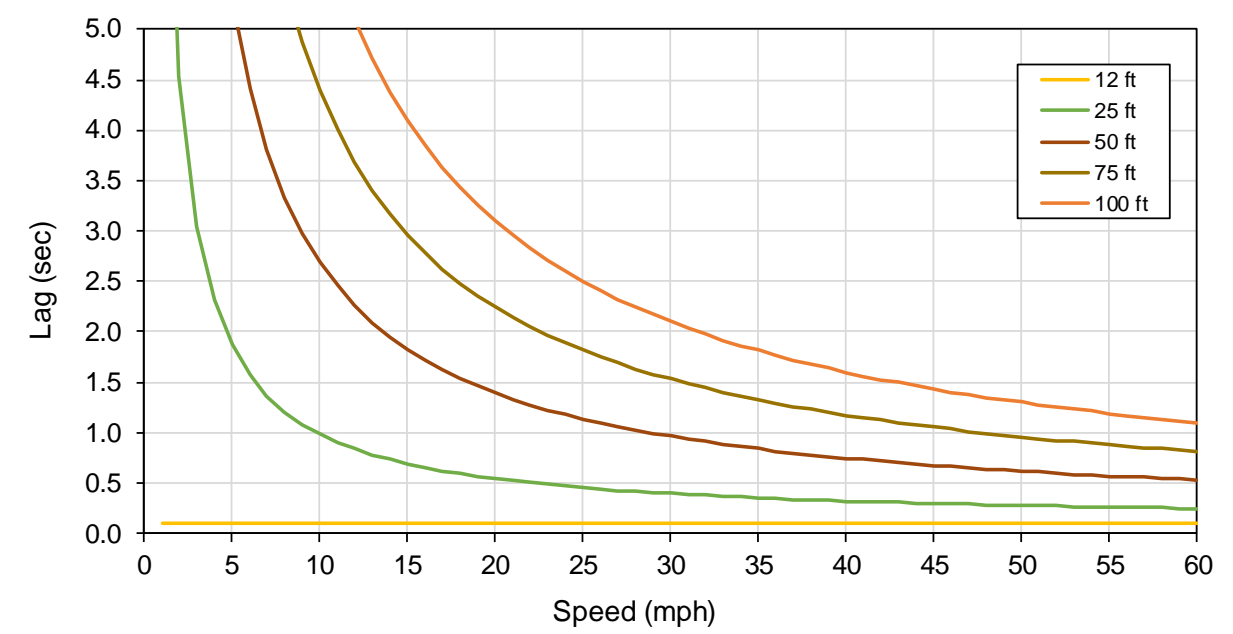

a. Hypothetical.

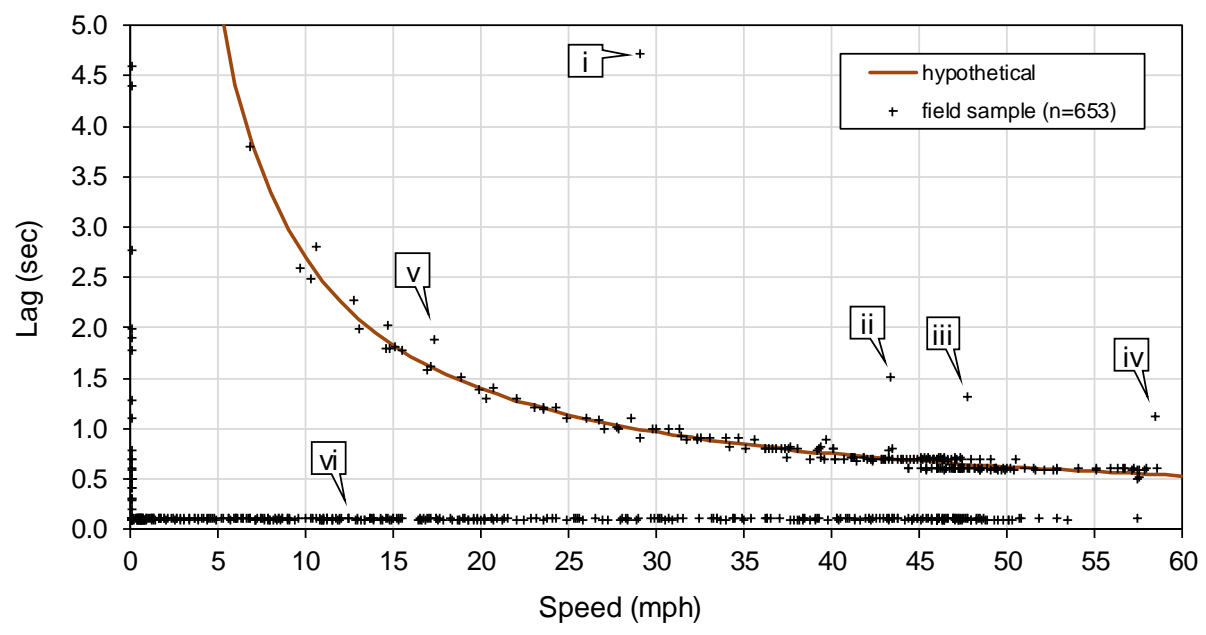

b. 50 foot spacing.

Figure 3. Performance of different waypoint spacings using 6 foot radius.

For this study, 50ft spacing is used which gives an estimated lag time of $0.59 \mathrm{~s}$ between matches at $55 \mathrm{mph}$. Figure $3 \mathrm{~b}$ shows the hypothetical lag curve and field samples collected, which performs reasonably close to the estimating function. Callout $i$ is an instance where three waypoints are missed by the vehicle when the vehicle is not centered in the lane. Callout ii and callout iii are two instances where four BSM messages are dropped each, therefore missing a portion of one waypoint. Callout iv is an instance where one waypoint is missed by the vehicle completely and callout $\mathrm{v}$ is when the vehicle veered slightly to the edge of the lane at low speed. Callout vi shows the matches within one waypoint which has a lag equivalent to the DSRC transmission interval of $0.1 \mathrm{~s}$. At faster speeds beyond $50 \mathrm{mph}$, the number of matches within one waypoint drops off. Overall $83 \%$ of the samples have lower lag than the hypothetical curve and $95 \%$ of the samples are within $5 \%$ or less. 


\section{Phase Calling using BSM}

On a valid match between a vehicle's location and a waypoint, a call is placed for an associated phase using the NTCIP phase call control object (29). To record only instances where the mainline phases $(\varnothing 2$ and $\varnothing 6)$ are called by the BSM, they must be separated from the loop detector calls to the same phase. Since NTCIP does not allow calling of detector channels directly, two "dummy" phases ( $\varnothing 9$ and $\varnothing 11)$ of 0.1 s duration are created on a third and fourth timing ring that each calls a dummy detector channel. Each of the dummy detector channels then calls the true movement phase.

By default, when a phase using call control is set, the call latches for a deterministic amount of time defined by the unit backup time parameter. For this study, $1 \mathrm{~s}$ is set for this parameter. To prevent the dummy phases from resting, buffer phases (Ø10 and Ø12) with backup prevent and recall enabled are programmed in each ring of the dummy phases, also with a $0.1 \mathrm{~s}$ duration.

Using the above described phase and detector programming, the BSMs are able to extend the green on a mainline phase when they are matched with a waypoint up to 1,050ft in advance of the stop bar.

\section{Terminating phases before a vehicle enters the dilemma zone}

This study proposes the use of Force Gap Out (FGO) to selectively early-terminate mainline phases before a subject vehicle enters the dilemma zone. FGO is triggered when it is determined that a $\mathrm{CV}$ will be within dilemma zone limits at the onset of yellow.

Dilemma zone performance deteriorates steeply during peak periods due to max outs occurring when one or more vehicles are within the dilemma zone. Sharma et al. proposed adjusting simultaneous gap out logic by turning off detectors at key times to improve performance (16). The dilemma zone limits defined by Parsonson's method is set between $10 \%$ and $90 \%$ on a probability of stopping curve (4), and is where max out is to be avoided. A binary regression model is used for estimating stopping distance and time within these thresholds (13), defined by:

$$
\frac{1}{1+e^{-\alpha-\beta_{1} V-\beta_{2} X}}
$$

where $V$ is the velocity and $X$ is the stopping distance. The parameters for heavy vehicles are used with $\alpha=0.1, \beta_{1}=-0.1$ and $\beta_{2}=0.08$. The estimated dilemma zone boundary is within 2.2s.

Figure 4 illustrates the probability of stopping curve defined by Eq. 1 for $55 \mathrm{mph}$. Between $10 \%$ and $90 \%$ of drivers choose to stop from $269 \mathrm{ft}$ to $449 \mathrm{ft}$ in advance of the stop bar when the onset of yellow occurs in this boundary (callout i, callout ii). Within these limits no FGOs would be triggered. At $50 \%$ probability of stopping (callout iii), the risk of conflict is the highest due to the same number of drivers wanting to stop as wanting to proceed (17).

The top horizontal axis indicates the amount of green time left in the phase, and the onset of yellow is aligned to the $90 \%$ threshold, corresponding to the $449 \mathrm{ft}$ mark. Any CV estimated to arrive at or after this point but before the $269 \mathrm{ft}$ mark ( 2.2 seconds later) would trigger FGO early from $449 \mathrm{ft}$ 


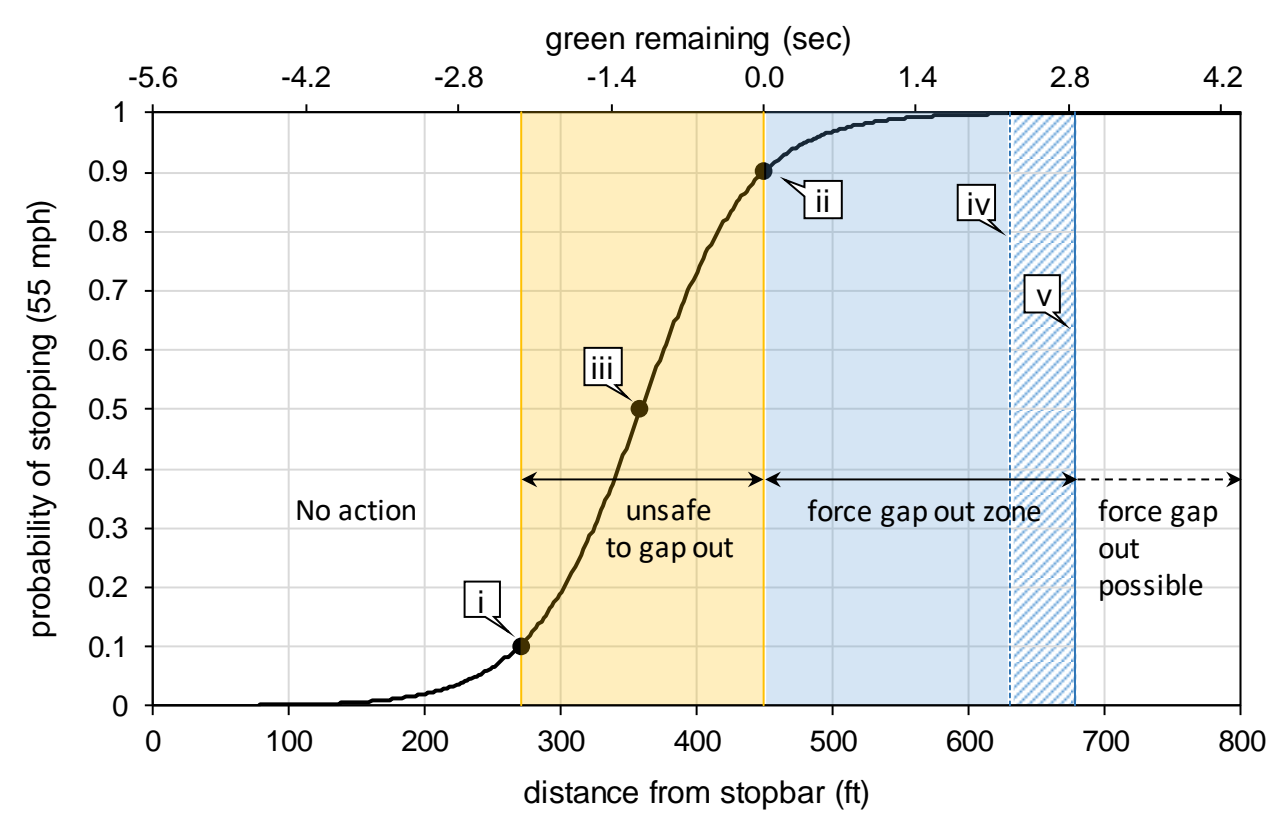

Figure 4. Identifying forced gap out zone for heavy vehicles.

to the $629 \mathrm{ft}$ mark (2.2s earlier, callout iv). An extra 0.59s is added to this limit (callout v) to account for any delay due to lag between waypoints defined in the previous section. Any FGO occurring in advance of this point is possible but may not be efficient.

In general, the FGO logic is triggered when the following conditions are met:

1. There is a call on any side-street movement;

2. The max green time remaining $t_{\text {max }}$ on phase $P$ is less than a critical threshold $\gamma_{\mathrm{c}}(2.2 \mathrm{~s}$ at $55 \mathrm{mph}$ );

3. Phase $P$ is currently on;

4. Phase $P$ is called by a $\mathrm{CV}$.

Once all of the above conditions are met, an alternative detection plan is enacted immediately to gap out all mainline phases. The logic is then blocked for $t_{d}$ seconds, set to the yellow and all-red clearance interval plus the min green of the next phase. After $t_{d}$ seconds, the detection is reverted to the original plan.

\section{IMPLEMENTATION}

FGO logic is implemented at US-231 and County Road 500 South in Indiana. Figure 5 describes a single trial run with FGO triggering from the mainline northbound phase. For the experiment, $\gamma_{c}$ is relaxed to 10 s to accommodate the difficulty of timing the test $\mathrm{CV}$ to enter the dilemma zone at speed during the critical time window. In addition, the mainline detection is set to constantly call using detector faulting so that the test can be run during the off-peak period when few vehicles would be affected. 


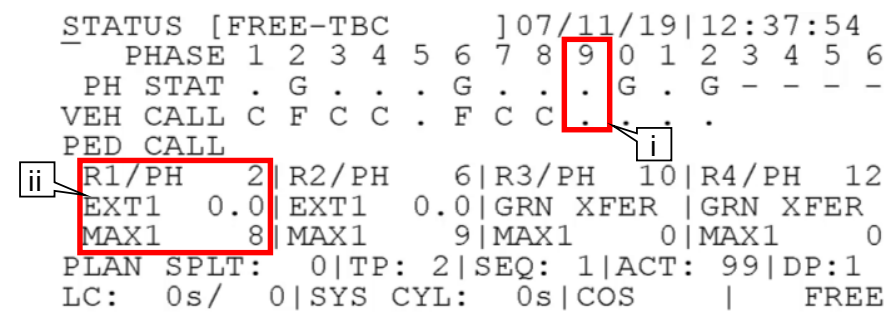

a. Before FGO at 12:37:54.

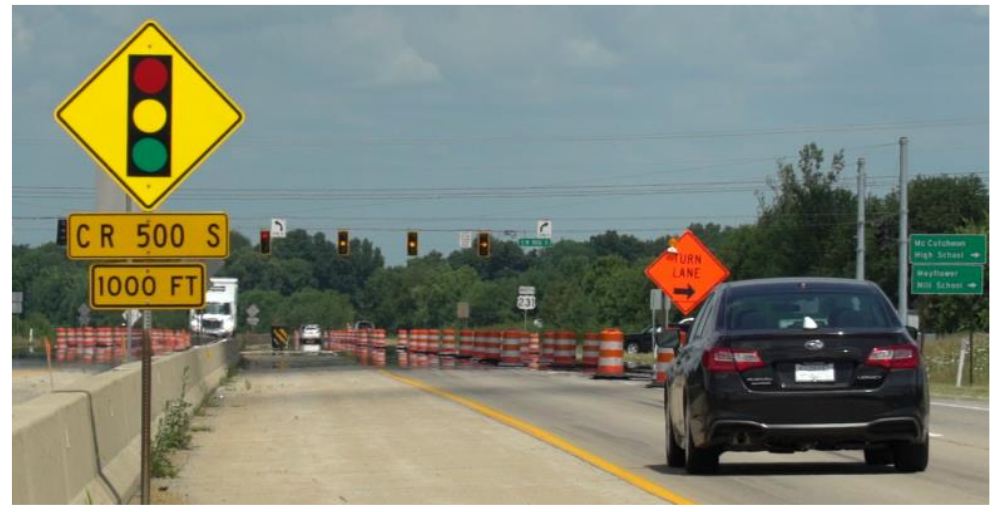

b. Looking towards intersection at 1000 foot mark just after FGO.

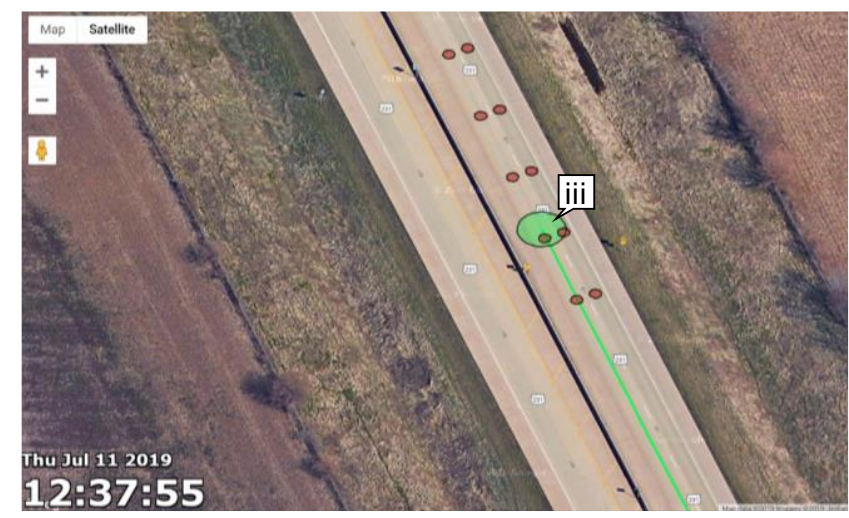

c. User interface.

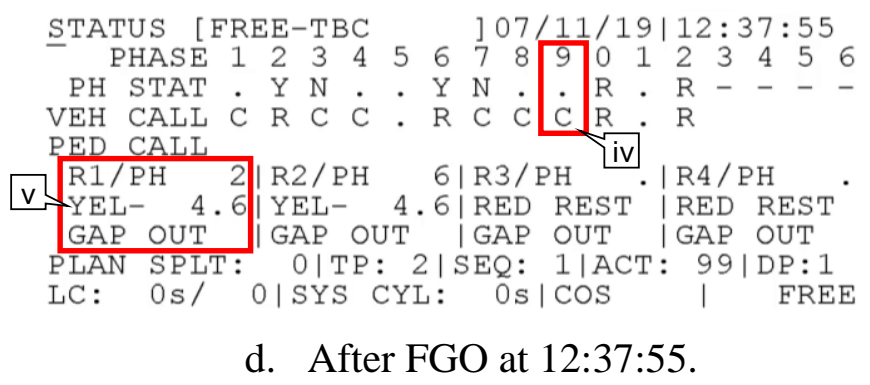

Figure 5. Field testing. 
Figure 5a shows the status screen of the traffic signal controller one second prior to FGO. Callout i shows the dummy $\varnothing 9$ not yet called as the CV is still upstream of the waypoint furthest from the stop bar. Callout ii shows the max timer for ring 1 is within the critical threshold. The test CV enters the waypoint area past the 1,000ft mark (Figure 5b), noting the stopped semi-truck in the opposing left-turn. The CV's location can be seen hitting two waypoints from the user interface (Figure 5c, callout iii). The green circle represents the location of the vehicle with the size exaggerated for visibility and the red circles represent the location of the virtual waypoints. Figure $5 \mathrm{~d}$ shows the instance when the FGO is triggered with a call on dummy $\varnothing 9$ (callout iv) and the resulting gap out (callout v).

\section{EVALUATION}

Although a CV may prevent itself from a dilemma zone incursion using FGO, it may cause other vehicles to be in the dilemma zone (where they would not have been) as an effect - likely during high volume periods. To determine if FGO reduces dilemma zone incursions overall within a period, a tradeoff estimation is made using ATSPM data.

Figure 6a through c illustrates the result of the estimate for the study intersection over a four month period. An FGO is considered in the estimation if a mainline phase terminated with a max out and any detection at the advance loop, interpolated to the onset of yellow time, falls within the dilemma zone limits. Figure 6a shows the number of FGO estimated in the northbound direction over a 24 hour period with the number of vehicles prevented from dilemma zone incursion and the number caused in both directions. At the northbound approach, most hours would break even or see slightly more vehicles prevented from a dilemma zone incursion than it would have caused, with the exception of the peak times of 06:00, 08:00, 16:00, and 17:00.

The southbound direction shows a greater benefit (Figure 6b), as more vehicles would be prevented from a dilemma zone incursion or at least break even for all hours. Overall, there are 310 vehicles (if equipped with OBUs) estimated to trigger FGO from March 1 through June 30, 2019, with 61 vehicles prevented in the northbound direction and 249 vehicles prevented in the southbound direction. The number of dilemma zone incursions caused by the FGOs is 173 in the northbound direction and 52 in the southbound direction for a total of 225 FGO-caused incursions. In terms of tradeoffs, the northbound direction had no change and the southbound direction has a net dilemma zone incursion reduction of $34 \%$.

The evaluation is likely a conservative estimate because it considers all caused dilemma zone incursions as heavy vehicles. However in field implementation and considering the mode distribution ( $26 \%$ heavy vehicles) at this location, the vehicle caused to be in the dilemma zone would likely be a passenger vehicle and would be able to stop more quickly than the heavy vehicle causing the FGO. In the future, higher penetration rate of CV would allow the logic to be customized to disable FGO in the case that a potentially caused vehicle is a heavy truck.

\section{CONCLUSIONS}

The rate of fatalities at signalized intersections involving heavy vehicles is nearly five times higher than for passenger vehicles. This study proposed using Connected Vehicle (CV) technology to trigger Force Gap Out (FGO) when a vehicle was expected to arrive within the dilemma zone limit 


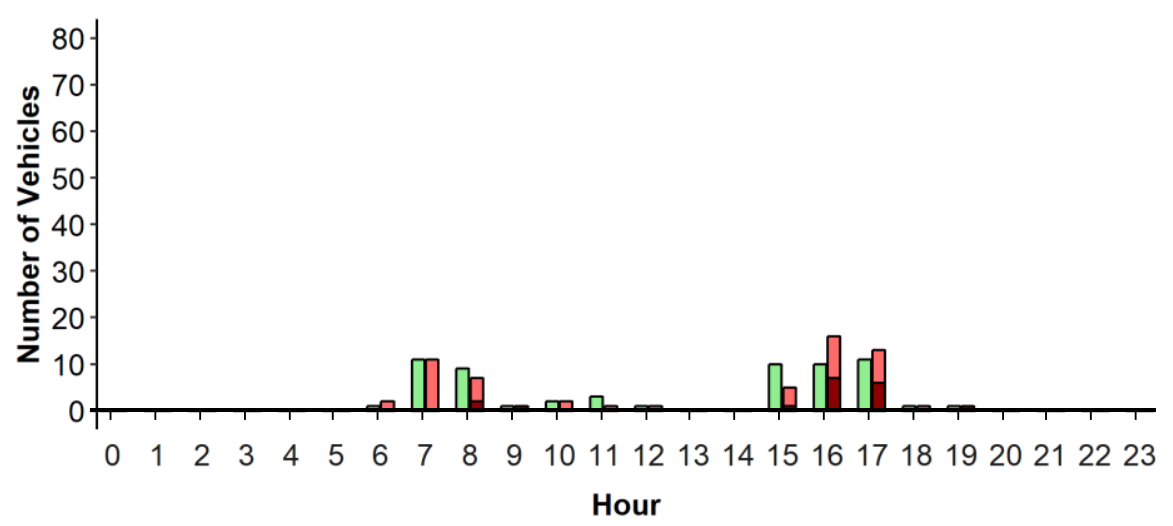

$\square$ Prevented $\square$ NB_Caused $\square$ SB_Caused

a. FGO triggered from northbound.

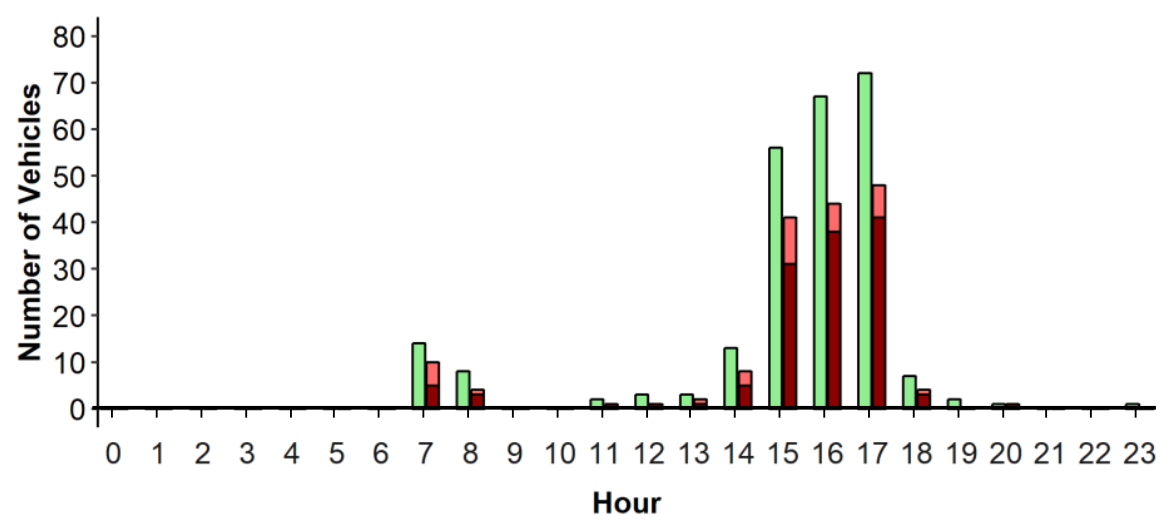

$\square$ Prevented $\square$ NB_Caused $\square$ SB_Caused

b. FGO triggered from southbound.

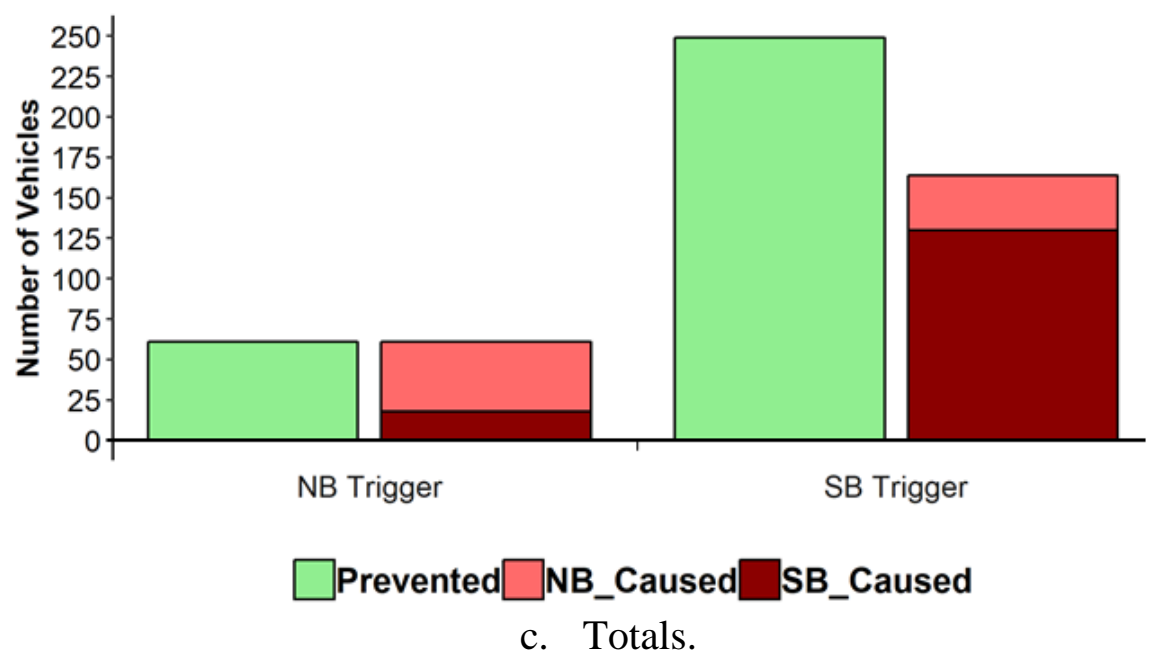

Figure 6. Dilemma zone performance, March 1, 2019 through June 30, 2019. 
at max out time at a fully-actuated intersection. The method leverages position data from Basic Safety Messages (BSMs) to map-match virtual waypoints. For a $55 \mathrm{mph}$ approach, it was determined that using a $6 \mathrm{ft}$ waypoint radius at 50ft spacings would be sufficient to match $95 \%$ of BSM data within a $5 \%$ lag threshold of 0.59s. Using Automated Traffic Signal Performance Measures (ATSPMs), it was estimated that dilemma zone incursions would break even for the northbound approach and be reduced by a net of $34 \%$ for the southbound approach.

A modification of the map-matching process can make use of consecutive pairs of BSM messages to construct $\mathrm{CV}$ trajectories. Alternative to matching points to points, FGO can be triggered when trajectories intersect with cross-sectional lines upstream of an approach to eliminate match inaccuracies when vehicles drift away from the center of a lane. Ideally features that track CV trajectories would be built into the next generation of traffic signal controllers. Another adjustment that would improve FGO is taking into account the actual speed of the approaching CV, which is already contained in the BSM, and adjust the critical threshold based on that speed to determine if FGO is necessary. Further investigations into FGO performance for different demand patterns, approach geometries, as well as taking into account penetration rates is recommended for future research.

\section{ACKNOWLEDGEMENTS}

This work was supported by the Joint Transportation Research Program and the Indiana Department of Transportation. The contents of this paper reflect the views of the authors, who are responsible for the facts and the accuracy of the data presented herein, and do not necessarily reflect the official views or policies of the sponsoring organizations. These contents do not constitute a standard, specification, or regulation.

\section{AUTHOR CONTRIBUTION}

The authors confirm contribution to the paper as follows:

(a) Study conception and design: Tom Platte, Howell Li, Darcy Bullock

(b) Data collection: Howell Li, Jijo Mathew, Tom Platte, Ben Smith, Enrique SaldivarCarranza

(c) Analysis and interpretation of results: Howell Li, Jijo Mathew, Tom Platte, Ben Smith

(d) Draft manuscript preparation: Howell Li, Jijo Mathew, Darcy Bullock

All authors reviewed the results and approved the final version of the manuscript.

\section{REFERENCES}

1. Intersection Safety, Federal Highway Administration. https://safety.fhwa.dot.gov/intersection/conventional/signalized/. Accessed Jul. 29, 2019.

2. Signalized Intersections: An Informational Guide, Federal Highway Administration. https://safety.fhwa.dot.gov/intersection/conventional/signalized/fhwasa13027/ch2.cfm\#s2 22. Accessed Jul. 29, 2019.

3. Vehicular Crash Data - Datasets - the Indiana Data Hub. https://hub.mph.in.gov/dataset/aries-crash-data-2007-2017. Accessed Jul. 29, 2019.

4. Parsonson, P. S. Signalization of High-Speed, Isolated Intersections. 1978.

5. Bonneson, J., K. Zimmerman, and M. Brewer. Engineering Countermeasures to Reduce 
Red-Light-Running. 2002.

6. Zegeer, C. V, and R. C. Deen. Green-Extension Systems at High-Speed Intersections. 1978.

7. Zimmerman, K. Additional Dilemma Zone Protection for Trucks at High-Speed Signalized Intersections. Transportation Research Record: Journal of the Transportation Research Board, Vol. 2009, No. 1, 2007, pp. 82-88. https://doi.org/10.3141/2009-11.

8. Gazis, D., R. Herman, and A. Maradudin. The Problem of the Amber Signal Light in Traffic Flow. Operations Research, Vol. 8, No. 1, 1960, pp. 112-132. https://doi.org/10.1287/opre.8.1.112.

9. Olson, P. L., R. Rothery, and S. Research. Driver Response to Amber Phase of Traffic Signals. Highway Research Board, No. Bulletin 330, 1962, pp. 40-51.

10. Sheffi, Y., and H. Mahmassani. A Model of Driver Behavior at High Speed Signalized Intersections. Transportation Science, Vol. 15, No. 1, 1981, pp. 50-61. https://doi.org/10.1287/trsc.15.1.50.

11. Chang, M.-S., C. J. Messer, and A. J. Santiago. Timing Traffic Signal Change Intervals Based on Driver Behavior. Transportation Research Record, Vol. 1027, 1985, pp. 20-30.

12. Bonneson, J. A., and K. H. Zimmerman. Effect of Yellow-Interval Timing on the Frequency of Red-Light Violations at Urban Intersections. Transportation Research Record, Vol. 1865, No. 1, 2004, pp. 20-27. https://doi.org/10.3141/1865-04.

13. Archer, J., and W. Young. Signal Treatments to Reduce Heavy Vehicle Crash-Risk at Metropolitan Highway Intersections. Accident Analysis \& Prevention, Vol. 41, No. 3, 2009, pp. 404-411. https://doi.org/10.1016/J.AAP.2008.12.015.

14. van der Horst, R. Driver Decision Making at Traffic Signals. Transportation Research Record, No. 1172, 1988.

15. Urbanik, T., A. Tanaka, B. Lozner, E. Lindstrom, K. Lee, S. Quayle, S. Beaird, S. Tsoi, P. Ryus, D. Gettman, S. Sunkari, K. Balke, and D. Bullock. Signal Timing Manual - Second Edition. Transportation Research Board, Washington, D.C., 2015.

16. Sharma, A., D. M. Bullock, and S. Peeta. Limitations of Simultaneous Gap-Out Logic. Transportation Research Record: Journal of the Transportation Research Board, Vol. 1978, No. 1, 2006, pp. 42-48. https://doi.org/10.1177/0361198106197800107.

17. Sharma, A., D. Bullock, and S. Peeta. Estimating Dilemma Zone Hazard Function at High Speed Isolated Intersection. Transportation Research Part C: Emerging Technologies, Vol. 19, No. 3, 2011, pp. 400-412. https://doi.org/10.1016/J.TRC.2010.05.002.

18. Bryant, C. W., H. A. Rakha, and I. El-Shawarby. Study of Truck Driver Behavior for Design of Traffic Signal Yellow and Clearance Timings. Transportation Research Record: Journal of the Transportation Research Board, Vol. 2488, No. 1, 2015, pp. 62-70. https://doi.org/10.3141/2488-07.

19. Sharma, A., D. M. Bullock, S. Velipasalar, M. Casares, J. Schmitz, and N. Burnett. Improving Safety and Mobility at High-Speed Intersections with Innovations in Sensor Technology. Transportation Research Record: Journal of the Transportation Research Board, Vol. 2259, No. 1, 2011, pp. 253-263. https://doi.org/10.3141/2259-24.

20. Ayoub, N. A. New Detection Technology Eliminates Dilemma Zones. 2014.

21. Emmelmann, M., B. Bochow, and C. C. Kellum. Vehicular Networking. 2010.

22. Intelligent Transportation Systems, ITS Deployments, CV Pilot Deployment Program, WYDOT Update at the Application Design Stage Webinar Q\&A. https://www.its.dot.gov/pilots/wydot_webinar_qa.htm. Accessed Jul. 29, 2019.

23. SAE International. J2735D: Dedicated Short Range Communications (DSRC) Message Set 
Dictionary $^{T M}$ - SAE International. Warrendale, PA, 2016.

24. Li, P., M. Abbas, and R. Pasupathy. A Stochastic Dilemma Zone Protection Algorithm Based on the Vehicles' Trajectories. Journal of Intelligent Transportation Systems, Vol. 19, No. 2, 2015, pp. 181-191. https://doi.org/10.1080/15472450.2014.977043.

25. Zha, L., Y. Zhang, P. Songchitruksa, and D. R. Middleton. An Integrated Dilemma Zone Protection System Using Connected Vehicle Technology. IEEE Transactions on Intelligent Transportation Systems, Vol. 17, No. 6, 2016, pp. 1714-1723. https://doi.org/10.1109/TITS.2015.2490222.

26. Feng, Y., M. Zamanipour, K. L. Head, and S. Khoshmagham. Connected Vehicle-Based Adaptive Signal Control and Applications. Transportation Research Record: Journal of the Transportation Research Board, Vol. 2558, No. 1, 2016, pp. 11-19. https://doi.org/10.3141/2558-02.

27. Njobelo, G., T. Sando, S. Sajjadi, E. Mtoi, E. E. Ozguven, and J. Sobanjo. Safety Evaluation of the Advanced Stop Assist System in Connected Vehicle Environment. Transportation Research Record: Journal of the Transportation Research Board, Vol. 2672, No. 22, 2018, pp. 47-57. https://doi.org/10.1177/0361198118797831.

28. Yang, X. (Terry), G.-L. Chang, Z. Zhang, and P. (Taylor) Li. Smart Signal Control System for Accident Prevention and Arterial Speed Harmonization under Connected Vehicle Environment. Transportation Research Record: Journal of the Transportation Research Board, 2019, p. 036119811983724. https://doi.org/10.1177/0361198119837242.

29. Object Definitions for Actuated Traffic Signal Controller (ASC) Units. NTCIP 1202:2005 V02.19. 2005.

30. Smaglik, E., A. Sharma, D. Bullock, J. Sturdevant, and G. Duncan. Event-Based Data Collection for Generating Actuated Controller Performance Measures. Transportation Research Record: Journal of the Transportation Research Board, Vol. 2035, 2007, pp. 97106. https://doi.org/10.3141/2035-11.

31. Day, C. M., D. M. Bullock, H. Li, S. M. Remias, A. M. Hainen, R. S. Freije, A. L. Stevens, J. R. Sturdevant, and T. M. B. Jr. Performance Measures for Traffic Signal Systems: An Outcome-Oriented Approach. Purdue University, West Lafayette, IN, 2014.

32. Sturdevant, J. R., T. Overman, E. Raamot, R. Deer, D. Miller, H. Li, A. Hainen, and S. M. Remias. Indiana Traffic Signal Hi Resolution Data Logger Enumerations. 2012.

33. EDC-4: Automated Traffic Signal Performance Measures (ATSPMs) | Federal Highway Administration. https://www.fhwa.dot.gov/innovation/everydaycounts/edc_4/atspm.cfm. Accessed Jul. 29, 2019.

34. Du, Y., M. Chowdhury, M. Rahman, K. Dey, A. Apon, A. Luckow, and L. B. Ngo. A Distributed Message Delivery Infrastructure for Connected Vehicle Technology Applications. IEEE Transactions on Intelligent Transportation Systems, Vol. 19, No. 3, 2018, pp. 787-801. https://doi.org/10.1109/TITS.2017.2701799.

35. Karney, C. F. F. Algorithms for Geodesics. 2012. 